\title{
Mechanical Behavior of Aluminum Phosphide under Pressure
}

\author{
S. DAOUD* \\ Laboratoire Matériaux et Systèmes Electroniques (LMSE), \\ Université Mohamed Elbachir El Ibrahimi de Bordj Bou Arreridj, Bordj Bou Arreridj, 34000, Algeria
}

(Received October 7, 2017; in final form November 9, 2017)

With the help of structural parameters and elastic constants obtained previously in our work (S. Daoud, N. Boiud, N. Lebga, J. Optoelectron. Adv. Mater. 16, 207 (2014)), different empirical formulae were successfully used to investigate: equation of state, the isotropic shear modulus, the Young modulus, the Cauchy ratio, the Born ratio, the Poisson ratio, the Pugh ratio, the Kleinman parameter, and the converse piezoelectric coefficient of the aggregate AlP material with cubic zinc-blende structure under pressure up to experimental pressure of phase transition $(9.5 \mathrm{GPa})$. In addition, the Debye temperature at equilibrium volume was predicted, the result obtained is in excellent agreement compared to the experimental ones, the deviation is less than $1.4 \%$.

DOI: 10.12693/APhysPolA.133.23

PACS/topics: AlP semiconductor, equation of state, mechanical behavior, piezoelectric coefficient

\section{Introduction}

To provide a more comprehensive understanding of IIVI and III-V tetrahedrally coordinated semiconductors, which have many applications, especially in the field of linear and nonlinear optics, solar energy conversion, lightemitting diodes, laser diodes and integrated optical devices [1]. In recent years many researches [1-15] have been carried out on phase transition, structural parameters, and other physical properties of these materials.

Using pseudopotential plane wave approach in the framework of density functional theory (DFT) method, Aouadi et al. [3] have studied structural, elastic, electronic and dynamical properties in both zinc-blende and nickel-arsenide (B8) phases of aluminum phosphide material. They found that all the elastic constants increase with application of pressure, whereas, both, the Born effective charges and the high frequency dielectric constant decrease with increase of hydrostatic pressure.

The structural parameters and electronic properties under pressure effect of AlP material in cubic zinc-blende phase have been studied by Jappor et al. [4], they used a semiempirical method, and they found that their calculated results are in good agreement with the experimental data. They conclude also the possibility of using their model in qualitative study of some other materials.

To investigate elastic properties, sound velocity and the Debye temperatures in zinc-blende III-P (BP, AlP, $\mathrm{GaP}$ and $\mathrm{InP}$ ) compounds under pressure effect, Bouhemadou et al. [5] have used the full-potential augmented plane wave plus local orbitals (FP-APW + lo) method. They have found a linear dependence of the bulk modulus and elastic constants versus applied pressure.

Under the effect of external pressure, the cubic zincblende phase of AlP compound transforms to the nickel-

*e-mail: salah_daoud07@yahoo.fr arsenic (NiAs) phase at about $9.5 \pm 5$ GPa [6]. Ameri et al. [7] used an $a b$ initio calculation in the framework of the density functional theory to study the effect of aluminum concentration on some physical properties of $\mathrm{Al}_{x} \operatorname{In}_{1-x} \mathrm{P}$ alloy.

To provide a more comprehensive understanding of AlP material, some interesting mechanical and thermal properties under pressure have been calculated and analyzed. The equation of state (EOS), the isotropic shear modulus, the Young modulus, the Cauchy ratio, the Born ratio, the Kleinman parameter, the Poisson ratio, the Pugh ratio, and the converse piezoelectric coefficient up to experimental pressure of phase transition $(9.5 \mathrm{GPa})$ of the aggregate material are predicted.

\section{Theoretical, results and discussions 2.1. Equation of state}

The understanding of EOS plays a very important role in both experimental and theoretical high pressure phase transition investigations of materials, because the study of EOS allows scientists to know more information on the nature of solid state theories and helps us to determine the values of several thermodynamic parameters [16]. Therefore, to study the structural parameters under pressure, the Vinet equation of state is usually used. The Vinet $[17,18]$ form of EOS was given by following expression [16-18]:

$$
\ln H=\ln B_{0}+1.5\left(B_{0}^{\prime}-1\right)(1-X) .
$$

Here $X=\left(V_{n}\right)^{1 / 3}$, and $V_{n}=V_{p} / V_{0}$ is the normalized volume, where $V_{p}$ is the volume at a value of no null pressure $P$, and $V_{0}$ is the volume at zero-pressure. The function $H$ is given as follows [16-18]:

$$
H=P X^{2} / 3(1-X) \text {. }
$$

The universal EOS curve of AlP tetrahedrally coordinated semiconductor was depicted in Fig. 1. As observed in Fig. 1, a linear correlation between $\ln H$ and $(1-X)$ is established. As far as we know, there is no data available in the literature on the universal EOS curve for this semiconductor. 


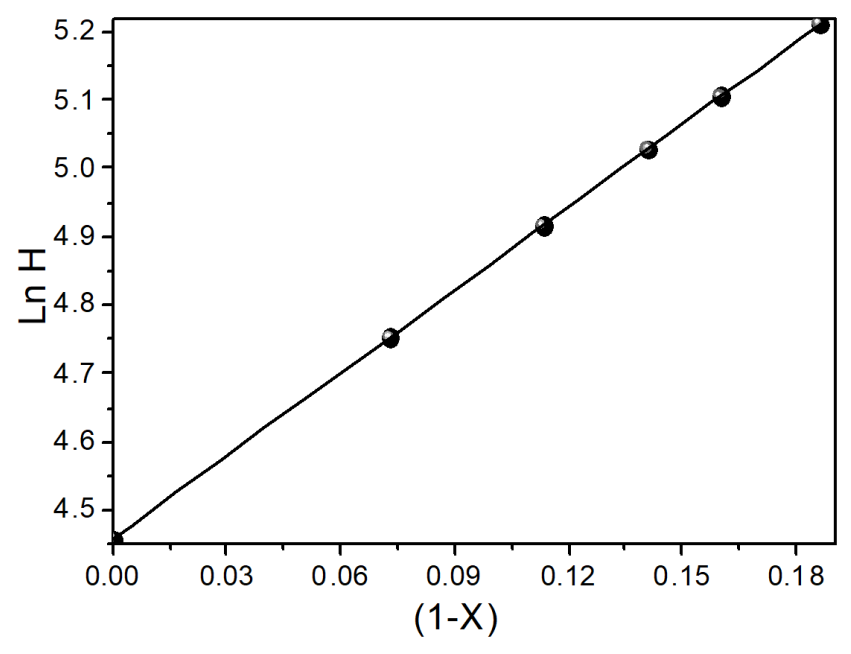

Fig. 1. Universal EOS of cubic zinc-blende AlP semiconductor expressed in the Vinet form.

\subsection{Mechanical properties}

2.2.1. Isotropic shear modulus, the Pugh ratio and the Poisson ratio

It should be noted that the elastic properties of semiconductors play a very important role in physics of solid state matter, because they are linked directly with many other physical quantities, especially: the heat capacity, the Debye temperature, the thermal expansion, and the Grüneisen parameter [19]. In some times, the semiconductor compounds are studied as aggregate polycrystalline materials, so the mean values of different quantities are usually used. The isotropic shear modulus $G$ is an average of two limits, named the Voigt modulus $G_{\mathrm{V}}$, and the Reuss modulus $G_{\mathrm{R}}$, it is expressed as [5]:

$$
G=\left(G_{\mathrm{V}}+G_{\mathrm{R}}\right) / 2 .
$$

For cubic crystals, these two quantities are related directly to the second order elastic stiffness constants $C_{i j}$; these two quantities are given as [8]:

$$
\begin{aligned}
& G_{\mathrm{V}}=\left(C_{11}-C_{12}+3 C_{44}\right) / 5, \\
& G_{\mathrm{R}}=\left[5\left(C_{11}-C_{12}\right) C_{44}\right] /\left[4 C_{44}+3\left(C_{11}-C_{12}\right)\right] .
\end{aligned}
$$

From the formula of Eqs. (4), it is important to note that the aggregate Voigt shear modulus $G_{\mathrm{V}}$ is more influenced by the sheared elastic constant $C_{44}$; whereas the aggregate Reuss shear modulus $G_{\mathrm{R}}$ is influenced almost equally by the sheared elastic quantity $\left(C_{11}-C_{12}\right)$ and $C_{44}$, respectively. The evaluated isotropic shear modulus $G$, the Voigt modulus $G_{\mathrm{V}}$ and the Reuss modulus $G_{\mathrm{R}}$ at zero-pressure are: 50.04, 45.08 and $47.56 \mathrm{GPa}$, respectively. We observe that our calculated isotropic shear modulus is relatively lower than the previous theoretical data (59.1 GPa) reported in Ref. [5]. It is acknowledged also that the isotropic shear modulus $G$ is used sometimes to give information about the Vickers hardness $H v$, where $G$ and $H v$ are varied in direct proportional manner. Therefore, a higher value of $G$ indicates a higher value of $H v$ and vice versa. Figure 2 shows the vari- ations of isotropic shear modulus $G$, the Voigt modulus $G_{\mathrm{V}}$ and the Reuss modulus $G_{\mathrm{R}}$ with hydrostatic pressure for tetrahedrally coordinated AIP material. We observe a quadratic dependence in all curves. All these three quantities of interest decrease monotonically with raising pressure.

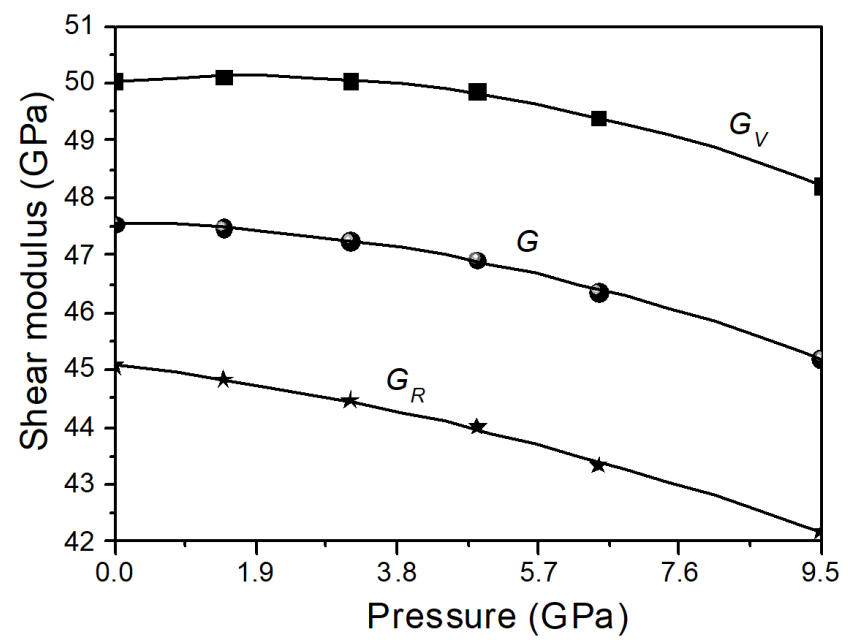

Fig. 2. Isotropic shear modulus, Voigt modulus and Reuss modulus of B3 AlP versus pressure.

Three analytical relations for the pressure dependence isotropic shear modulus $G$, the Voigt modulus $G_{\mathrm{V}}$ and the Reuss modulus $G_{\mathrm{R}}$ are given by the following quadratic fit, i.e.:

$$
\begin{aligned}
& G_{\mathrm{V}}=50.04+11.01 \times 10^{-2} p-3.18 \times 10^{-2} p^{2}, \\
& G_{\mathrm{R}}=45.08-14.83 \times 10^{-2} p-1.66 \times 10^{-2} p^{2}, \\
& G=47.56-1.90 \times 10^{-2} p-2.42 \times 10^{-2} p^{2} .
\end{aligned}
$$

Our obtained results regarding the pressure derivatives at zero pressure namely: $\partial G_{\mathrm{V}} / \partial P, \partial G_{\mathrm{R}} / \partial P$ and $\partial G / \partial P$ of AlP semiconductor are: $0.11,-0.14$, and -0.02 , respectively. Unfortunately, to the best of our knowledge, there are no data available in the literature on the pressure derivatives of $G, G_{\mathrm{V}}$, and $G_{\mathrm{R}}$ for this semiconductor.

The ductility and brittleness are two probable characteristics of semiconductor materials, which are related directly to the elastic constants [13]. Pugh [20] proposed a ratio to study the nature of the mechanical behavior in materials, it is named Pugh's ratio which is the rapport between the bulk modulus $B$ and the isotropic shear modulus $G$. The value 1.75 is considered as limit to separate the ductile and the brittle domains. A high value of $B / G$ indicates tendency of material for ductility behavior, while a low value of $B / G$ indicates its tendency for brittleness behavior. At zero pressure, the ratio $B / G$ of AlP is equal to 1.89 , it is more than 1.75 ; this result suggests that this compound is prone to ductile behavior. It is observed that our value (1.89) of $B / G$ is relatively higher than the previous theoretical value (1.54) reported by Varshney et al. [21]. The reason of this big difference is perhaps due to the different formulae used to predict the 
values of the shear modulus $G$, where the average value between the Voigt modulus $G_{\mathrm{V}}$ and the Reuss modulus $G_{\mathrm{R}}$ is used here, while only $G_{\mathrm{V}}$ was used in the work of Varshney et al. [21].

Another elastic quantity which was also proposed to study the nature of mechanical behavior of material, is the Poisson ratio $\sigma$, it is related to the bulk modulus $B$ and the isotropic shear modulus $G$ by the following expression: $\sigma=(3 B-2 G) /(6 B+2 G)[22]$. In Fig. 3, the Pugh ratio and Poisson ratio are plotted against pressure. As shown in Fig. 3, we found that both Pugh's and the Poisson ratios increase almost linearly with increase of pressure, indicating that AlP tetrahedrally coordinated material is prone to ductile manner in the range of pressure 0-9.5 GPa.

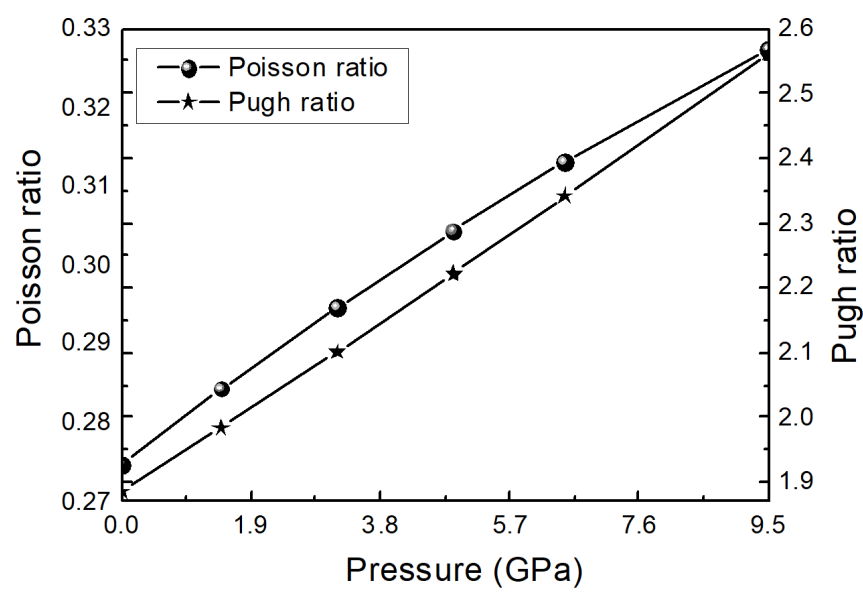

Fig. 3. The Pugh ratio and the Poisson ratio of B3 AlP semiconductor versus pressure.

We observe also that our value at zero pressure of the Poisson ratio $(\approx 0.27)$ is relatively higher than the previous theoretical data $(\approx 0.23)$ reported by Bouhemadou et al. [5], and is relatively lower than the previous theoretical data (0.335) reported by Wang and Ye [23]; so our result is localized between these two theoretical values.

2.2.2. Young modulus, Cauchy and Born ratios, and Kleinman parameter

We further attempt to determine the functional derivatives as Young modulus E, Cauchy ratio Ca, Born ratio Bo, and the Kleinman parameter $\zeta$. The Young modulus $E$ is one of important mechanical parameter; for the isotropic material (aggregate polycrystalline material), it can be determined with the help of the following equation [5]:

$$
E=9 B G /(3 B+G) \text {. }
$$

Contrarily to the Poisson ratio, our calculated value of $E$ is esteemed at about $121 \mathrm{GPa}$, it is relatively lower than the previous theoretical data $(145.6 \mathrm{GPa})$ reported by Bouhemadou et al. [5], and it is relatively higher than the previous theoretical data $(87.8 \mathrm{GPa})$ reported by Wang and Ye [23]. Therefore, our result of Young modulus is also localized between these two theoretical values. To predict the variation of the $\mathrm{Ca}$ and $\mathrm{Bo}$ as function of pressure up 9.5 GPa of AlP material, the different relationships given in Refs. [24, 25] are used here. The predicted values of the Young modulus E, Ca and Bo ratios up to $9.5 \mathrm{GPa}$ are reported in Table I, and compared with available data of the literature $[5,21,23,26]$.

TABLE I

The Young modulus $(E)$, Cauchy ratio $(\mathrm{Ca})$ and Born ratio (Bo) of $\mathrm{AlP}$ in pressure range from 0 to $9.5 \mathrm{GPa}$.

\begin{tabular}{c|c|c|c}
\hline \hline$P[\mathrm{GPa}]$ & $E[\mathrm{GPa}]$ & $\mathrm{Ca}$ & Bo \\
\hline 0.00 & 121.26 & 1.10 & 1.09 \\
& $145.6[5]$ & $0.915[21]$ & $2.67[21]$ \\
& $87.8[23]$ & $1.05[26]$ & $0.86[26]$ \\
1.46 & 121.95 & 1.17 & 1.09 \\
3.17 & 122.38 & 1.25 & 1.10 \\
4.87 & 122.43 & 1.34 & 1.10 \\
6.50 & 121.78 & 1.42 & 1.11 \\
9.50 & 120.01 & 1.58 & 1.10
\end{tabular}

At zero pressure, our calculated values of $\mathrm{Ca}$ and $\mathrm{Bo}$ are esteemed at about 1.10 and 1.09, respectively. Our value (1.10) of the Cauchy ratio $\mathrm{Ca}$ is in good agreement with the result (1.05) reported by Adachi [26], the deviation is less than $5 \%$. Our value (1.09) of the Born ratio (Bo) is relatively higher than the previous one (0.86) reported by Adachi [26], and is relatively lower than the result (2.67) of Varshney et al. [21]. It is important to note that the value 2.67 of Bo reported by Varshney et al. [21] is incorrect. Thus it is perhaps due to the incorrect formula used by Varshney et al. [21] to esteem this quantity. By using the numerical values: 225, 130, and $142 \mathrm{GPa}$ of the elastic constants $C_{11}, C_{12}$, and $C_{44}$ respectively used by Varshney et al. [21], the correct value of the Bo is 1.69 which is much lower than the incorrect value (2.67). Our result of Bo is also localized between these two values $(0.86$ and 1.69$)$ of the literature.

It is very important to note that our values $(\approx 1.1)$ of Bo are almost invariable as a function of the pressure. This behavior was also observed in the case of the binary GaAs and AlAs and the ternary $\mathrm{Al}_{x} \mathrm{Ga}_{1-x} \mathrm{As}$ semiconducting materials [27], where the value of about 1.1 was also found, and it was found almost invariable as a function of the concentration. Since the Bo is correlated with the bond ionicity in II-VI, III-V, and I-VII semiconducting compounds [28], we can clearly observe that the measured ionicities of AlP and GaAs are also almost the same (0.307 for AlP and 0.310 for GaAs) [28]. For AlP semiconductor in its zinc-blende structure, the internal strain parameter (named also the Kleinman parameter) and the elastic constants are related by the following expression [5]:

$$
\zeta=\left(C_{11}+8 C_{12}\right) /\left(7 C_{11}+2 C_{12}\right) .
$$

In Fig. 4, the internal strain parameter $\zeta$ is plotted versus pressure. As shown in Fig. 4, we found that $\zeta$ increases quadratically with increase of pressure up to $9.5 \mathrm{GPa}$. It is to be noted that a low value of $\zeta$ signifies a large resis- 
tance against bond bending or bond angle distortion and high value of $\zeta$ means a small resistance against bond bending or bond angle distortion in solid [29].

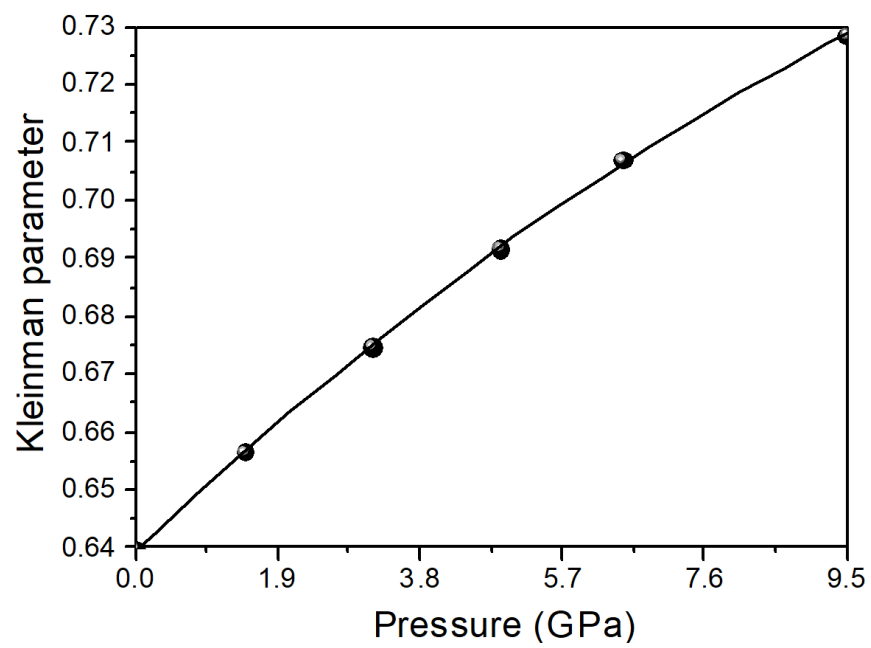

Fig. 4. Internal strain parameter of B3 AlP semiconductor versus pressure up to $9.5 \mathrm{GPa}$.

The internal strain parameter $\zeta$ begins with a value of 0.64 at zero pressure, and it reaches a value about 0.73 at $9.5 \mathrm{GPa}$. We observe that our calculated value (0.64) of $\zeta$ at zero-pressure is in excellent agreement with the previous theoretical data (0.631) obtained by Bouhemadou et al. [5], the deviation between the two values is only about $1.4 \%$. It is important to note that the internal strain parameter $\zeta$ of AlP tetrahedrally coordinated material is also reported by Wang and Ye [23]; the deviation between our calculated value $(0.64) \zeta$ and the value (0.64) obtained by Wang and Ye [23] is about $6 \%$.

\subsection{Piezoelectric coefficient}

In the case of $B 3$ structure, the piezoelectric tensors in both direct and converse piezoelectric effects contain only one constant. In first one, this constant is usually called $e_{14}$, whereas in the second one it is called $d_{14}[30]$. The converse piezoelectric coefficient $d_{14}$ is related to the elastic constant $C_{44}$, and the direct piezoelectric coefficient $e_{14}$ by the following formula [31]:

$$
d_{14}=e_{14} / C_{44} \text {. }
$$

Attempting to predict the effect of hydrostatic pressure on the converse piezoelectric coefficient $d_{14}$ of AlP tetrahedrally coordinated compound, the different results are presented in Fig. 5. As shown in Fig. 5 the converse piezoelectric coefficient increases gradually with pressure, where it begins with a value of $0.8 \mathrm{pm} / \mathrm{V}$ at zero pressure, and it reaches a value of about $4.7 \mathrm{pm} / \mathrm{V}$ at $9.5 \mathrm{GPa}$. However, to the best of our knowledge, no theoretical or experimental data of the converse piezoelectric coefficient $d_{14}$ of AlP compound have appeared anywhere in literature. So, future experimental works or other theoretical calculations will contribute for the validating of our calculated results of this quantity.

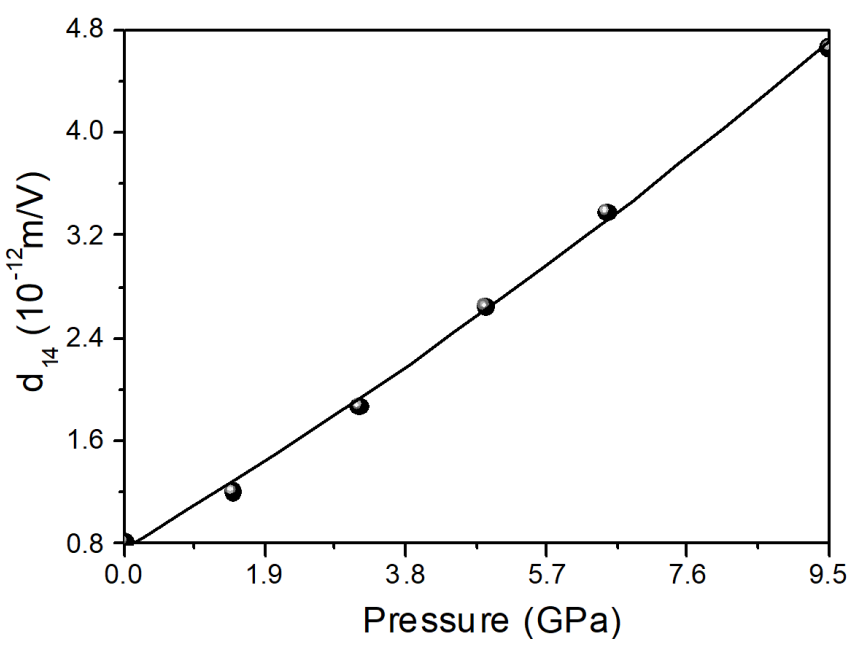

Fig. 5. Converse piezoelectric coefficient $d_{14}$ of $B 3 \mathrm{AlP}$ semiconductor versus applied pressure.

\subsection{Debye temperature}

The Debye temperature of solids can be obtained from the elastic constants and one from the specific heat measurements [32]. For several materials with cubic structure, the Debye temperature $\theta_{D}$ can be obtained from the elastic constants $C_{i j}$ by means of the following formula [33]:

$$
\begin{aligned}
\theta_{D}^{3} & =\frac{3.15}{8 \pi}\left(\frac{h}{k}\right)^{3}\left(\frac{n}{g^{3 / 2} v_{a}}\right)\left(C_{11}-C_{12}\right)^{1 / 2} \\
& \times\left(C_{11}+C_{12}+2 C_{44}\right)^{1 / 2}\left(C_{44}\right)^{1 / 2},
\end{aligned}
$$

where $h$ is the Planck constant, $k$ is the Boltzmann constant, $n$ is the number of atoms in unit cell of volume $v_{a}$, and $g$ is the density.

The predicted value of the Debye temperature is reported in Table II, and compared with available data of the literature [5, 34, 35].

\section{TABLE II}

The Debye temperature of AlP, in comparison of our value with available theoretical $[5,34]$ and experimental [35] data.

\begin{tabular}{c|c|c}
\hline \hline & our & other \\
\hline$\theta_{\mathrm{D}}[\mathrm{K}]$ & 596 & $603[5] ;$ from 479 to $673[34] ; 588[35]$ exp.
\end{tabular}

It is seen that our calculated equilibrium Debye temperature $\theta_{\mathrm{D}}$ of $\mathrm{AlP}$ tetrahedrally coordinated is in very good agreement with the previously calculated data [5, 34] and experimental value reported in Ref. [35]. The value $596 \mathrm{~K}$ obtained by us deviates from the experimental value $588 \mathrm{~K}$ reported in Ref. [35] with only about $1.36 \%$. The value $596 \mathrm{~K}$ obtained by us deviates from the theoretical one $603 \mathrm{~K}$ reported by Bouhemadou et al. [5] with only about $1.16 \%$. 


\section{Conclusion}

Using different empirical formulae, the mechanical behavior, piezoelectric coefficient, and Debye temperature of aluminum phosphide material under pressure up to experimental pressure of phase transition $(9.5 \mathrm{GPa})$ were determined. All these quantities are predicted with the help of the structural parameters and elastic constants obtained by using first-principle calculations published previously in our paper [11]. The isotropic shear modulus, the Young modulus, the Cauchy ratio, the Born ratio, the Poisson ratio, the Pugh ratio, the Kleinman parameter and the converse piezoelectric coefficient of the aggregate AlP material are investigated. The Debye temperature is also successfully predicted and analyzed, where the deviation between our result $(596 \mathrm{~K})$ and the experimental one $(588 \mathrm{~K})$ is only about $1.36 \%$.

At zero pressure, our results are in good agreement with the experimental and other theoretical results. On the basis of the analysis of Pugh's and the Poisson ratios, the AlP material behaves as ductile manner.

\section{References}

[1] D.S. Yadav, C. Kumar, J. Singh, Parashuram, G. Kumar, J. Eng. Comput. Innov. 3, 26 (2012).

[2] S. Labidi, H. Meradji, S. Ghemid, S. Meçabih, B. Abbar, J. Optoelectron. Adv. Mater. 11, 994 (2009).

[3] S. Aouadi, P. Rodriguez-Hernandez, K. Kassali, A. Muńoz, Phys. Lett. A 372, 5340 (2008).

[4] H.R. Jappor, M.A. Abdulsattar, A.M. Abdullettif, Open Condens. Matter Phys. J. 3, 1 (2010).

[5] A. Bouhemadou, R. Khenata, M. Kharoubi, T. Seddik, A.H. Reshak, Y. Al-Douri, Comput. Mater. Sci. 45, 474 (2009).

[6] R.G. Greene, H. Luo, A.L. Ruoff, J. Appl. Phys. 76, 7296 (1994)

[7] M. Ameri, A. Bentouaf, M. Doui-Aici, R. Khenata, F. Boufadi, A. Touia, Mater. Sci. Appl. 2, 729 (2011).

[8] S. Daoud, N. Bioud, N. Bouarissa, Mater. Sci. Semicond. Proc. 31, 124 (2015).

[9] A. Mujica, P. Rodríguez-Hernández, S. Radescu, R.J. Needs, A. Muńoz, Phys. Status Solidi B 211, 39 (1999).

[10] S. Daoud, Comput. Mater. Sci. 111, 532 (2016).

[11] S. Daoud, N. Bioud, N. Lebga, J. Optoelectron. Adv. Mater. 16, 207 (2014).

[12] H. Meradji, S. Drablia, S. Ghemid, H. Belkhir, B. Bouhafs, A. Tadjer, Phys. Status Solidi B 241, 2881 (2004).
[13] D. Varshney, G. Joshi, M. Varshney, S. Shriya, Solid State Sci. 12, 864 (2010).

[14] S. Daoud, A. Latreche, A. Bencheikh, Cryst. Res. Technol. 51, 115 (2016).

[15] M. Talati, P.K. Jha, Int. J. Mod. Phys. B 24, 1235 (2010).

[16] P. Bhardwaj, M. Sarwan, R. Dubey, S. Singh, J. Mol. Struct. 1043, 85 (2013).

[17] P. Vinet, J. Ferrante, J.R. Smith, J.H. Rose, Phys. Rev. B 35, 1945 (1987).

[18] P. Vinet, J. Ferrante, J.R. Smith, J.H. Rose, 'Universality in the Compressive Behavior of Solids, NASA Technical Memorandum 87303, N 86-26775, 1986.

[19] D. Varshney, S. Shriya, M. Varshney, R. Khenata, Comput. Mater. Sci. 61, 158 (2012).

[20] S.F. Pugh, Philos. Mag. 45, 823 (1954).

[21] D. Varshney, G. Joshi, N. Kaurav, R.K. Singh, J. Phys. Chem. Solids 70, 451 (2009).

[22] S. Stølen, R.G. Trønnes, Phys. Earth. Planet. In. 164, 50 (2007).

[23] S.Q. Wang, H.Q. Ye, Phys. Status Solidi B 240, 45 (2003).

[24] S. Adachi, Physical Properties of III-V Semiconductor Compounds, Wiley, New York 1992, p. 25.

[25] Processing and properties of compound semiconductors, in series Semiconductors and Semimetals, Vol. 73, Eds. R. Willardson, H.S. Nalwa, Academic Press, San Diego 2001, p. 27.

[26] S. Adachi, Properties of Group-IV, III-V and II-VI Semiconductors, Wiley, England 2005.

[27] S. Adachi, Properties of Aluminium Gallium Arsenide, The Institution of Electrical Engineers, London 1993, p. 25.

[28] V.P. Mikhal'chenko, Phys. Solid State 45, 453 (2003).

[29] K. Kim, W.R.L. Lambrecht, B. Segal, Phys. Rev. B 50, 1502 (1994).

[30] J. Yang, Special Topics in the Theory of Piezoelectricity, Springer, Science+Business Media, LLC, 2009.

[31] S. Daoud, N. Bioud, N. Lebga, Pramana J. Phys. 81, 885 (2013).

[32] B. Lüthi, Physical Acoustics in the Solid State, Springer-Verlag, Berlin 2005.

[33] M. Blackman, Philos. Mag. 42, 1441 (1951).

[34] V. Kumar, V. Jha, A.K. Shrivastava, Cryst. Res. Technol. 45, 920 (2010).

[35] Handbook of Chemistry and Physics, Ed. D.R. Lide, 80th ed., CRC Publication, 1999-2000. 